\title{
Heart Osteosarcoma Presenting as Infective Endocarditis: A Case Report of a Patient With a Cardiac Pacemaker and Triple Malignancies
}

\author{
Toivo Laks ${ }^{\mathrm{a}, \mathrm{h}}$, Katlin Kirik ${ }^{\mathrm{b}}$, Enn Joestec, Sigurd F. Lax ${ }^{\mathrm{d}, \mathrm{e}}$, Anita Liiver ${ }^{\mathrm{a}}$, Andrei Samarin , \\ Ljudmilla Kalinina $^{\mathrm{b}}$, Mai Puusepp ${ }^{\mathrm{f}}$, Toomas Sarev ${ }^{\mathrm{g}}$
}

\begin{abstract}
Primary and metastatic cardiac sarcomas represent rare neoplasms with a variable clinical course. We present a rare case of an 84-yearold man with a cardiac pacemaker and heart osteosarcoma, hepatocellular and prostatic carcinoma, who was admitted with suspected symptoms of infective endocarditis. Findings of cardiac osteosarcoma in a patient with a pacemaker and three malignancies have not been reported before in the literature.
\end{abstract}

Keywords: Primary and metastatic heart osteosarcoma; Cardiac pacemaker; Infective endocarditis; Triple malignancies

\section{Introduction}

Primary cardiac tumors are rare, with an incidence in early autopsy series from $0.001 \%$ to $0.3 \%[1,2]$. The majority of these are benign, primary malignant heart tumors accounting for less than 25\% [3]. Primary cardiac osteosarcomas are extremely rare and account for $<10 \%$ of primary malignant neoplasms of the heart [4-6]; and less than 50 cases have been reported

Manuscript submitted July 12, 2019, accepted August 6, 2019

aDepartment of Cardiology, North Estonia Medical Centre, 19 Sutiste, 13419 Tallinn, Estonia

bDepartment of Internal Medicine, North Estonia Medical Centre, 19 Sutiste, 13419 Tallinn, Estonia

'Department of Pathology, North Estonia Medical Centre, 19 Sutiste, 13419 Tallinn, Estonia

${ }^{\mathrm{d} D e p a r t m e n t ~ o f ~ P a t h o l o g y, ~ H o s p i t a l ~ G r a z ~ I I, ~ A c a d e m i c ~ T e a c h i n g ~ H o s p i t a l ~ o f ~}$ the Medical University Graz, Goestingerstrasse 22, AT-8020 Graz, Austria

eInstitute of Pathology and Molecular Pathology, Johannes Kepler University Linz, Linz, Austria

fDepartment of Radiology, North Estonia Medical Centre, 19 Sutiste, Tallinn, Estonia

gDepartment of Cardiology, Norfolk and Norwich University Hospital NHS Foundation Trust, Colney Lane, Norwich, NR4 7UY, Norfolk, UK

hCorresponding Author: Toivo Laks, Department of Cardiology, North Estonia Medical Centre, 19 Sutiste, 13419 Tallinn, Estonia.

Email: toivo.laks@regionaalhaigla.ee

doi: https://doi.org/10.14740/jmc3333 worldwide [7]. Most cardiac tumors are metastatic and 20 - 40 times more common than primary tumors $[4,8]$. Less than 30 cases of antemortem diagnosis of osteosarcoma metastasising to the heart and less than 100 cases of cardiovascular involvement have been described in the literature [9-12]. Although primary cardiac osteosarcoma could occur in any heart chamber, the vast majority of cases originated from the left atrium. Osteosarcomas metastatic to the heart most commonly involve the right-sided cardiac chambers $[13,14]$.

We report a sporadic case of an 84-year-old man with a pacemaker diagnosed with heart osteosarcoma, hepatocellular and prostatic adenocarcinoma and presenting with symptoms mimicking infective endocarditis.

The publication was approved by the Ethics Committee of the National Institute for Health Development of Estonia.

\section{Case Report}

An 84-year-old male was referred to the emergency department of North Estonia Medical Centre with a history of fever over 3 weeks, weakness, tachypnea (respiratory rate: 40/min) and a holosystolic precordial grade 4/6 murmur. Eight weeks earlier he had developed bradycardia and dizziness, and he underwent pacing generator replacement.

His past medical history includes two myocardial infarctions (30 and 15 years ago). A dual chamber rate adaptive (DDDR) pacemaker was implanted 11 years ago to manage his grade 3 atrioventricular (AV) block. He had been hypertensive for more than 30 years. Mild myocardial hypertrophy with inferior wall hypokinesia but normal left ventricular (LV) ejection fraction $(\mathrm{EF})$ were seen on transthoracic echocardiography (TTE) 1.5 years before along with left atrial (LA) dilatation and moderate mitral and aortic sclerosis with trivial regurgitation without stenosis and normal right-sided cavities.

Initial workup on admission included an electrocardiogram (ECG) that demonstrated regular atrial driven ventricular pacing. Laboratory signs of an acute infection with increased leukocyte count (white blood cell $(\mathrm{WBC}))\left(21 \times 10^{9} / \mathrm{L}\right)$ with marked neutrophilia (74\%) and raised C-reactive protein (CRP) $(106 \mathrm{mg} / \mathrm{L}$, summary in Table 1) were present. Chest X-ray demonstrated a round opacity measuring approximately $1 \mathrm{~cm}$ in the upper field of the left lung. Otherwise, the findings were normal. Pacing electrodes in the right chambers of 
Table 1. Laboratory Results

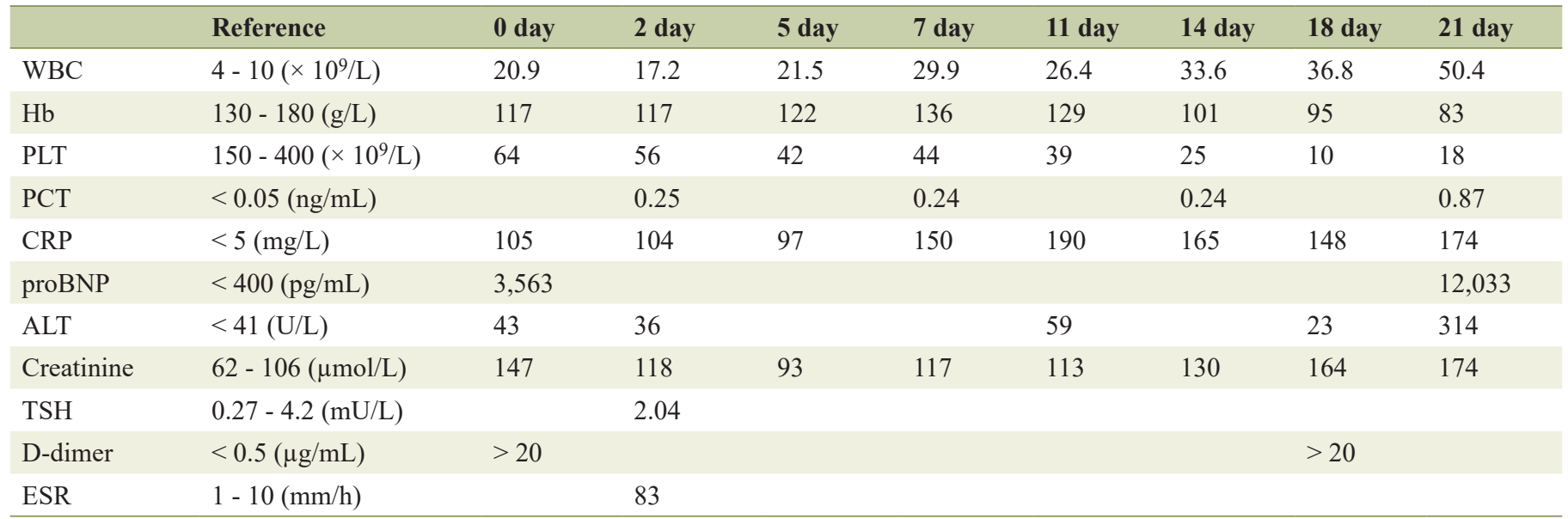

WBC: white blood cell; Hb: hemoglobin; PLT: platelet; PCT: procalcitonin; CRP: C-reactive protein; proBNP: pro b-type natriuretic peptide; ALT: alanine aminotransferase; TSH: thyroid stimulating hormone; ESR: erythrocyte sedimentation rate.

the heart were intact. Abdominal ultrasound showed a slightly inhomogeneous mass measuring $2.8 \times 2.4 \mathrm{~cm}$ in the lower part of the right lobe of the liver. Differential diagnoses included a metastasis or a primary tumor. Neither lymphadenopathy nor ascites was reported.

Whole body computed tomography (CT) was performed on the same day. The abdomen was scanned in native arterial and portal phase and thorax in arterial phase for pulmonary embolism. On unenhanced CT scan, the liver mass appeared isodense to liver parenchyma with smooth edges and bulging outside of the liver contours. There was no significant enhancement in the arterial phase, and the mass was slightly hypodense in the portal phase (Fig. 1a). The main trunk of the pulmonary artery (PA) and the right PA were dilated at $4 \mathrm{~cm}$ and $3.5 \mathrm{~cm}$ respectively. There was a large filling defect representing a thrombus in the upper lobe branch of the right PA and smaller filling defects in the lower lobe subsegmental branches of the right PA (Fig. 1b). A pulmonary nodule measuring 1 $\mathrm{cm}$ in diameter was detected in the upper lobe of the left lung, which was thought to be benign due to low CT density. No other pulmonary nodules or enlarged lymph nodes were found (Fig. 1c). Incidentally, a significant filling defect in the right atrium (RA) of the heart with subtotal obliteration of the appendage was found. The atrial pacing lead was attached to the RA appendage (RAA) next to the mass. The mass was thought to represent a thrombus (Fig. 1d).

On the two-dimensional (2D) TTE there was a mass seen in the RA with a dimension of $4.2 \times 2.0 \mathrm{~cm}$. Its structure was described inhomogeneous, with echogenicity of the soft tissue and without calcifications. The mass seemed to be attached to the lateral wall of the RA and surrounded the pacemaker electrode fixed in the RAA. Part of the mass was moving through the tricuspid valve orifice into the right ventricle (RV) during diastole but did not cause any obstruction (Fig. 2a, b). The function of both ventricles was normal. There was mild hypertrophy of the left ventricle, and both atria were slightly dilated. There were no signs of pulmonary hypertension or pericardial effusion. Based on the clinical findings and the findings of the
CT scan suspicion of the thrombotic mass was raised.

The history of recent pacing generator change, signs and symptoms of suspicion on acute infection or electrode endocarditis (fever, marked WBC and high CRP) justified commencing antibacterial therapy. Cefuroxime $4.5 \mathrm{~g} /$ day was given intravenously (IV). Due to marked thrombocytopenia $\left(66 \times 10^{9} / \mathrm{L}\right)$ enoxaparin was administrated in a reduced dose (6,000 U/day). The patient was clinically stable, and his blood cultures were negative. Notwithstanding this, fever, WBC, and CRP remained at the same level as on admission. Therefore, antibacterial treatment was switched on the fifth day (ampicillin + sulbactam $4.5 \mathrm{~g} /$ day IV and gentamicin $240 \mathrm{mg} /$ day IV). Fondaparinux (2.5 - $7.5 \mathrm{mg})$ was injected subcutaneously instead of enoxaparin due to a suspicion of heparin-induced thrombocytopenia. Despite these changes in treatment, he remained febrile for the next 5 days the temperature was between 38.2 and $38.5^{\circ} \mathrm{C}$. He also complained of pain in the whole body.

In the purpose of follow-up of the dynamics another TTE and transesophageal echocardiography (TEE) was performed 9 days after the first TTE. In the RA we found a multilocular mass with several interconnected immobile and mobile structures with soft tissue echogenicity attached to the lateral wall. The dimensions had increased up to $6.0 \times 2.6 \mathrm{~cm}$, and it was partly moving into the RV during diastole without obstructing the inflow to the tricuspid orifice (Fig. 2c, d). Besides, an immobile part of the mass of $5.5 \times 3.0 \mathrm{~cm}$ was seen in the RAA, which extended to the ostium of the superior vena cava (SVC) not obstructing the blood flow. The immobile mass in the RAA surrounded the pacing electrode without infiltrating the atrial wall or causing pericardial effusion. Furthermore, no vegetations were suspected on the cusps of the tricuspid valve and the pacemaker electrodes.

ECG-gated contrast-enhanced CT of the thorax was performed 10 days after the first whole-body CT to depict the mass in more detail. A large irregularly shaped soft tissue mass with signs of contrast enhancement in the portal phase, measuring $5.8 \times 4.4 \times 5.6 \mathrm{~cm}$ was found extending into the RAA. 

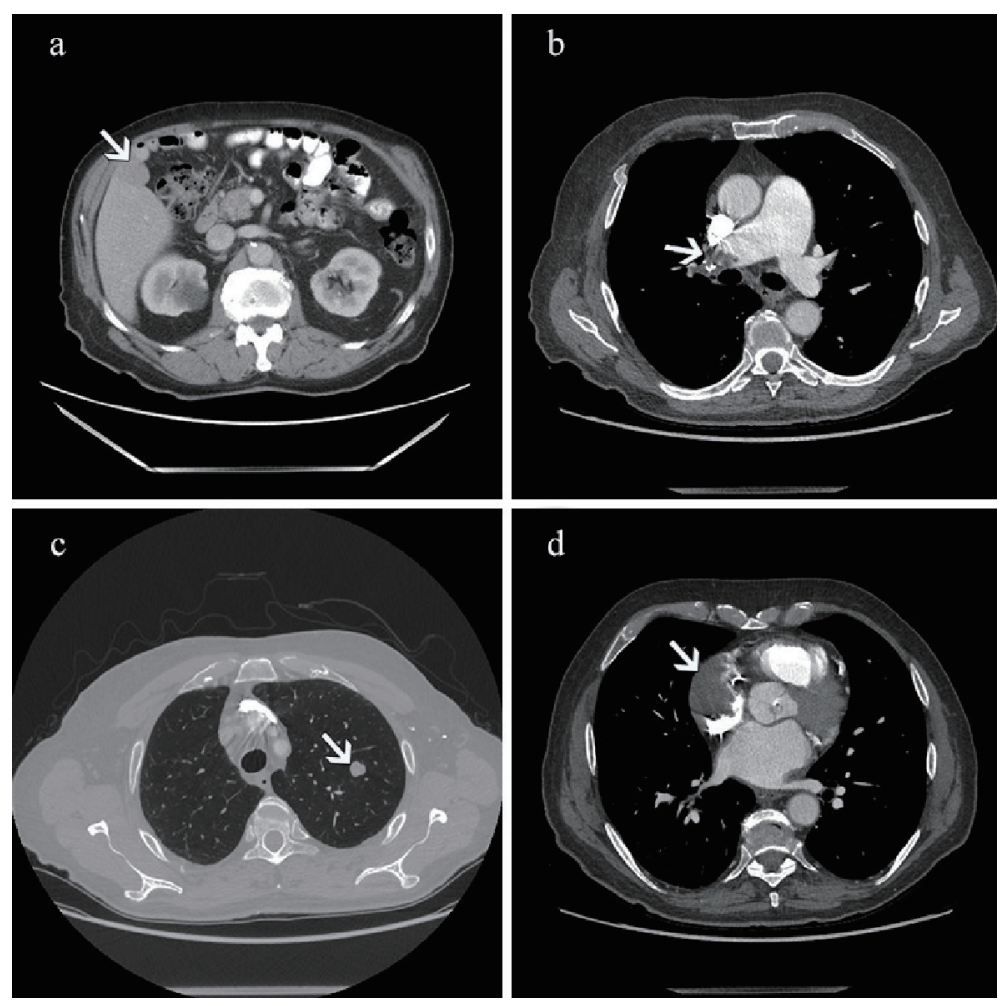

Figure 1. (a) Contrast-enhanced CT scan performed on the day of admission. The abdominal image acquired in portal venous phase shows a slightly hypodense bulging mass in the right lobe of the liver (arrow). (b) CT scan shows a large filling defect representing thrombus in the right pulmonary artery (arrow). There is an enlargement of the main pulmonary trunk and right pulmonary artery. (c) On CT scan there is a nodule measuring $1 \mathrm{~cm}$ in diameter in the upper lobe of the left lung (arrow). (d) CT scan shows a large filling defect in the right atrium (arrow) of the heart with subtotal obliteration of the appendage. CT: computed tomography.

Compared to the previous CT scan, the structure had grown in size, partially surrounding the atrial pacing lead. The second
$5.0 \times 2.2 \mathrm{~cm}$ mass was mobile, moving between the RA and RV.
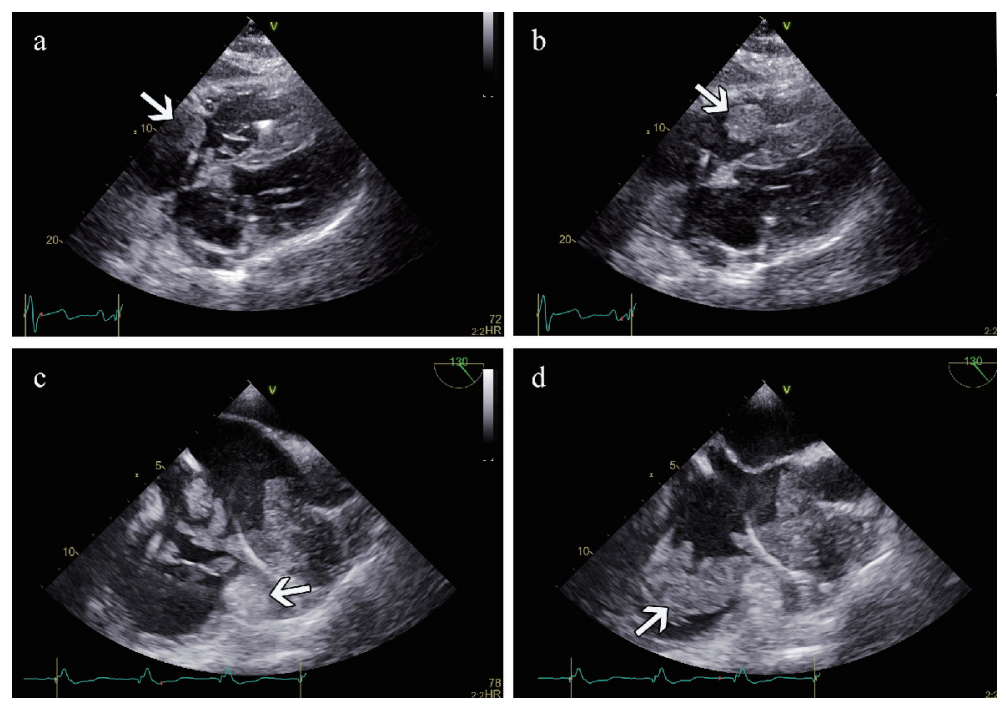

Figure 2. (a) TTE (subcostal view) in systole. A mass is detected in the right atrium (arrow). (b) TTE in diastole. The same mass is noticed in the right ventricular inflow tract (arrow). (c) TEE in systole. An immobile structure is demonstrated in the RAA (arrow) surrounding the pacemaker electrode. Mobile masses are demonstrated in the RA. (d) TEE in diastole. Mobile masses have moved into the RV inflow tract (arrow). TTE: transthoracic echocardiogram; TEE: transesophageal echocardiography; RAA: right atrial appendage. 
The ventricular pacing lead was separated from the mass. Malignant looking upper lobe nodule of the left lung had enlarged, and a small area of subpleural right lung consolidation had developed there as well. There was still a sizeable inhomogeneous density thrombus filling the upper lobe branch of the right PA, which raised the possibility of a tumor thrombus. The expansion in the right lobe of the liver also raised suspicion of a malignant process. Taking into account the potential metastatic nodule in the left lung, potentially malignant mass in the liver and enhancing mass in the RA of the heart, there was strong suspicion for metastatic disease with the potential primary tumor being in the liver. There were neither signs of infection in the area of the implanted pacemaker nor deep vein thrombosis on ultrasound.

Although the patient's high fever subsided only temporarily, $\mathrm{SatO}_{2}$ was $88 \%$ (with $\mathrm{O}_{2} 2 \mathrm{~L} / \mathrm{min}$ ), platelets (PLTs) dropped to $25 \times 10^{9} / \mathrm{L}$ (it was thought to be associated with the increased consumption), WBC and CRP did not decrease, and the patient remained clinically stable. Repeated blood cultures were negative. We could not exclude infection of the pacing electrodes. Therefore, we suspected that the structures could still be covered by thrombotic mass, which can result in an endocarditis-like clinical picture. Since the patient refused all invasive diagnostic procedures (including a biopsy), his conservative management was based on clinical symptoms. Piperacillin with tazobactam $4 \mathrm{~g} / 0.5 \mathrm{~g} /$ day IV was replaced after 4 days with imipenem and cilastatin $0.5 \mathrm{~g} / 0.5 \mathrm{~g} /$ day IV, and also vancomycin 2 - 3 g/day was given IV. Subcutaneous antithrombotic treatment was administered as well.

Four days later magnetic resonance imaging (MRI) of the abdomen was performed in purpose to characterise the hepatic lesion in detail. It was T1 isointense and T2 hyperintense with signs of marked diffusion restriction. We could not acquire contrast-enhanced series due to his inability to hold the breath. The mass showed a slight late enhancement in the delayed phase series. Additionally, a capsule-like peripheral enhancement was detected with characteristics not typical for a primary liver tumor. It was unclassifiable, possibly an atypical hemangioma. The differential diagnosis also included a hypovascular metastasis.

Three days later the patient's general condition destabilised, he complained of dyspnea, tachypnea, weakness, and melena developed. His $\mathrm{Hb}$ decreased to $95 \mathrm{~g} / \mathrm{L}$, PLT to $10 \times$ $10^{9} / \mathrm{L}, \mathrm{WBC}$ increased $\left(37 \times 10^{9} / \mathrm{L}\right)$. On gastroscopy at the large curvature, an ulcer $(0.7 \mathrm{~cm})$ covered with fibrin and surrounded with petechiae and a polyp $(0.3 \mathrm{~cm})$ were found. Despite the reduction of the dose of fondaparinux $(2.5 \mathrm{mg}$, subcutaneously) and infusion of red blood cells, PLTs and freshly frozen plasma the patient deceased 21 days after his admission.

Post mortem examination of the heart revealed a large white-greyish nodal mass in the RA with a diameter of $6.5 \mathrm{~cm}$, which was tightly fixed to the lateral wall. Tumor mass extended into the RAA and extended partially through the ostium of the tricuspid valve into the ventricle, not obstructing it. In the ostium of the SVC, a non-occlusive tumor $(2.0 \times 5.0 \mathrm{~cm})$ was found of the same consistency and color, covered with thrombotic mass. The RA pacing electrode, which was fixed to the appendage, was surrounded by a tumor and thrombotic masses. The ventricular pacing electrode was fixed in the api- cal septal area of the RV. Both electrodes were covered with thrombotic masses. Macroscopically and histologically there were no findings for endocarditis or vegetations. The LV was moderately hypertrophic, and the mitral annulus was calcified. Two post-infarct scars measuring up to $2.0 \mathrm{~cm}$ were found in the septal and posterolateral walls of the LV. Coronary tree revealed diffuse non-occlusive atherosclerotic plaque disease.

In the apical area of the left lung, there was a roundish grey nodule measuring $1.5 \mathrm{~cm}$. In the small branches of the pulmonary arteries, many small emboli (histologically confirmed as osteosarcomas) were found bilaterally. In the lower lobes of both lungs, there was partial atelectasis with 900 and $1,100 \mathrm{~mL}$ of pleural fluid respectively.

In the central part of the right hepatic lobe, a roundish pinkish-white tumor was found in subcapsular location consisting of some small nodes and measuring $3.5 \mathrm{~cm}$.

The prostate revealed a $4.5 \mathrm{~cm}$ yellow-pink honeycomblike lobulated nodule. The sigmoid colon demonstrated moderate diverticulitis. The abdominal or thoracic cavities were free of enlarged lymph nodes.

Histology was performed in the Departments of Pathology of North Estonia Medical Centre and the Hospital Graz II. The tumors in the heart (Fig. 3) and the lungs (Fig. 4a, b) had a myxoid appearance and consisted of the spindle and pleomorphic rhabdoid and sometimes signet-ring cells, which were immunohistochemically negative for cytokeratins (AE1/AE3), desmin and smooth muscle actin, but at least partially positive for osteonectin. Focally, osteoid-like material could be seen. In the sections from the heart, the tumor was found within the lumen, but it did not infiltrate the myocardium. The hepatic tumor revealed a solid and papillary architecture and consisted of polygonal cells with eosinophilic and clear cytoplasm and marked nuclear pleomorphism. Immunohistochemistry was positive for hepatocyte antigen and cytokeratins but negative for osteonectin (Fig. 4c). The histological diagnosis of a poorly differentiated (high-grade) sarcoma with osteogenic differentiation in the heart with metastasis to both lungs was established. The hepatic tumor was considered a poorly differentiated hepatocellular carcinoma. Sections from the prostate demonstrated a moderately differentiated acinar adenocarcinoma, Gleason score $6(3+3)$ (Fig. 4d).

\section{Discussion}

We describe a rare and unusual case of a patient with a cardiac pacemaker and osteosarcoma of the heart. Also, hepatocellular carcinoma and prostatic adenocarcinoma were detected.

Usually, diagnostic problems arise in the case of a previously undiagnosed tumor in the heart. Primary cardiac tumors are rare. Both primary and metastatic cardiac osteosarcomas are extremely rare, and less than 100 cases of antemortem diagnosis have been described altogether [7, 9-12]. Diagnosis can be more complicated in a patient with a cardiac pacemaker with a variety of diagnostic possibilities such as a thrombus, a caseous calcification of the valvular annulus, primary or metastatic tumor, abscess or endocarditis $[15,16]$.

In our case the fast and exact path to the diagnosis was 


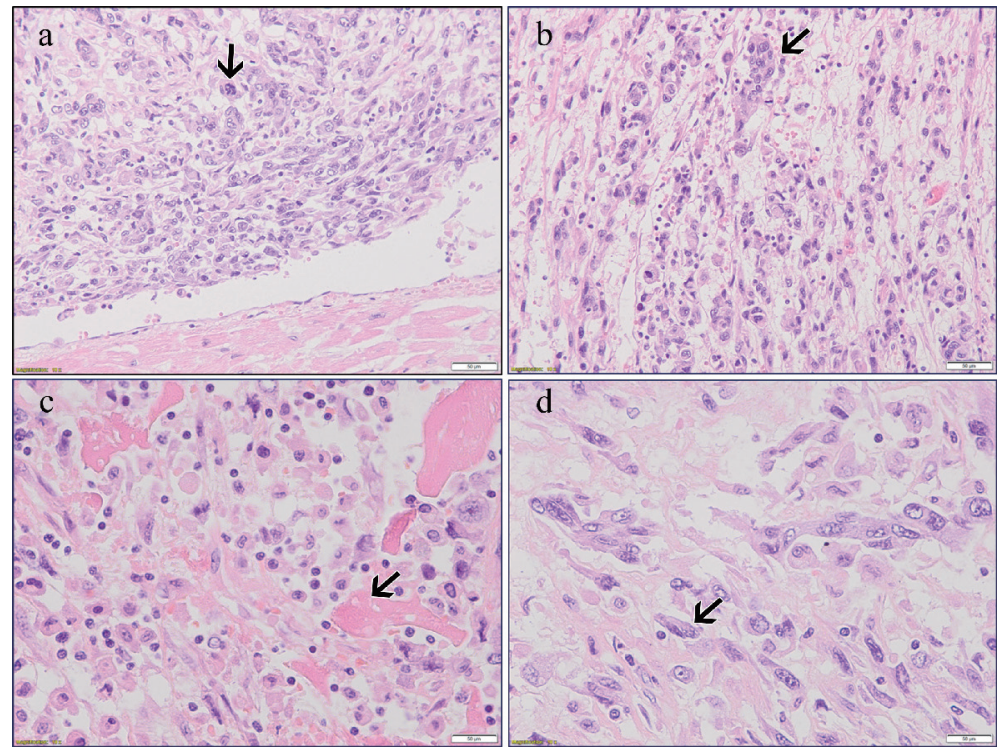

Figure 3. (a) Primary cardiac extraskeletal osteosarcoma. Pleomorphic tumor cells (arrow) and normal myocardium (H\&E, $\times 20)$. (b) Osteoclast-like giant cells (arrow) (H\&E, $\times 20)$. (c) Neoplastic osteoid matrix (arrow) (H\&E, × 20). (d) Giant and spindle cells (arrow) (H\&E, × 40).

complicated due to contradictions in history: recently changed pacing generator, the unusual clinical course of the disease simulating inflammation, tumor or thrombus surrounding the electrodes in the right heart, liver mass, nodules in the lung and filling defects in the pulmonary arteries. Since the clinical course was simulating pacemaker-endocarditis or a thrombus on the electrode, antibacterial treatment was initiated. Diagnostic alternatives considered were a primary malignant tumor in the liver with metastasis into the right side of the heart and to the lungs and a primary heart tumor with metastases to the lungs $[3,17,18]$. Since antibacterial treatment with different and combined antibiotics was ineffective, a strong suspicion of a malignant tumor (above all in liver) arose, with a concept based on earlier clinical observations that most of the metastases located in the right chambers of the heart $[13,14]$ and up to $3 \%$ of patients with carcinomas have cardiac metastases identified at autopsy. Liver malignancies are one of the most frequent tumors to spread directly through inferior vena cava [19]. Our patient did not complain of aching bones; clinically a tumor was not suspected, and a bone scan was not performed. Nevertheless, metastatic skeletal tumor thrombi most often from femur and humerus extending to the heart have been

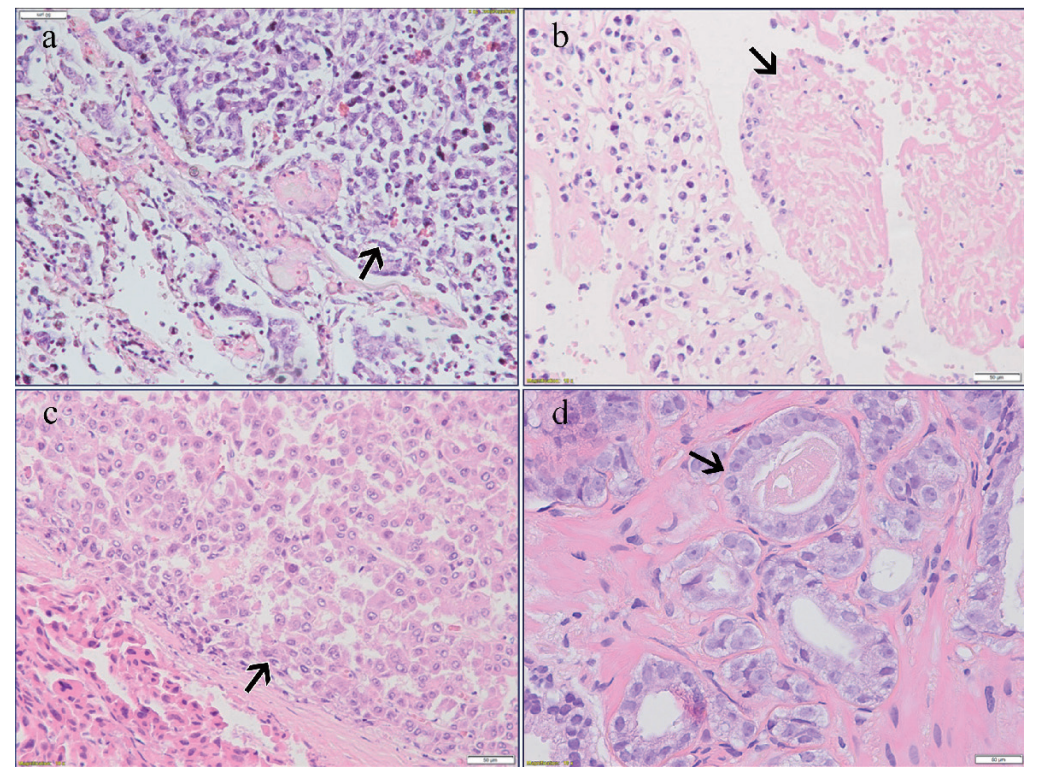

Figure 4. (a) Metastatic pulmonary osteosarcoma (arrow) $(H \& E, \times 20)$. (b) Tumor thrombus in the lung (arrow) $(H \& E, \times 20)$. (c) Hepatocellular carcinoma (arrow) $(H \& E, \times 20)$. (d) Acinar adenocarcinoma of the prostate (arrow) $(H \& E, \times 40)$. 
described [20-22]. In rare cases, extra-skeletal osteosarcoma may spread via the hematogenous route to an unusual cardiac localisation such as the LA $[11,14]$.

Primary cardiac osteosarcoma of the right side of the heart is extremely rare $[23,24]$. The clinical manifestations, regions of metastases (more than $50 \%$ to the lungs), imaging features, and laboratory tests are similar to other types of primary cardiac tumors $[17,25]$. Calcifications on imaging (CT) are crucial for differentiating osteosarcoma [26], but we encountered a case that was difficult to diagnose because imaging revealed no calcifications. The pathogenesis of primary cardiac osteosarcoma is not clear. It could develop through metaplasia of connective tissue or by malignant degeneration of embryonic somatic residues, which arise from undifferentiated mesenchymal stem cells in the myocardium and transform into active osteoblasts [23, 27]. Proposed etiological factors are a mechanical injury by causative agents and radiotherapy [28].

Furthermore, the heart is known to have a skeleton. Namely, fibrous trigone and annulus are the essential parts of it. Histologically the annulus has fibrotic and elastic fibres, and this structure is also in continuity with valvular leaflet tissue, the atrium and the ventricles. The hypothesis of the pathogenesis of cardiac osteosarcoma could be based on malignant metaplasia and transformation of fibrotic and elastic fibres, which makes up the trigone [29].

We also considered the opportunity that the undifferentiated cardiac tumor developed from metastatic hepatocellular carcinoma by dedifferentiation. We very carefully investigated both tumors histologically and by several immunohistochemical markers and did not find any similarity based on histomorphological and immunohistochemical features. Therefore, we concluded that it is more likely that the tumors in the heart and the liver are independent neoplasms, the former with pulmonary metastases. However, we did not perform molecular analyses to rule out a metastatic disease in the heart completely. Acinar adenocarcinomas of the prostate are common in advanced age and therefore, not an unexpected finding.

Multiple primary malignancies are common only in 3-5\% of tumors [30]. Despite its low incidence, the association of two malignancies in a single patient has been widely reported in the literature, while only a relatively small number $(0.5 \%)$ of three malignancies have been described including primary cardiac sarcomas [30-32]. Some cases of cardiac sarcomas (pleomorphic spindle cell sarcoma, angiosarcoma) in pacemaker patients are described $[16,33]$. At the same time, there are no clinical reports of patients (with or without a cardiac pacemaker) with primary osteosarcoma of the heart after double carcinomas of the liver and prostate. Only immunological studies have demonstrated that novel cytokine interleukin-32 (IL-32) could be involved in cancer development, including prostate, liver and heart (adenocarcinoma, hepatocellular carcinoma and osteosarcoma) [34].

\section{Conclusions}

We conclude that there is most likely a collision of two carcinomas: hepatocellular carcinoma and osteogenic sarcoma of the heart. The prostatic adenocarcinoma is a common finding in senior men. From the provided histology it is more likely that the tumors of the heart and the liver are independent. However, without molecular analysis (which was not performed) de-differentiation in a metastatic hepatocellular carcinoma cannot be excluded entirely. Injury of an electrode as a mechanical factor in the pathogenesis of cardiac osteosarcoma is not yet confirmed.

\section{Acknowledgments}

None to declare.

\section{Financial Disclosure}

None to declare.

\section{Conflict of Interest}

None to declare.

\section{Informed Consent}

Not applicable.

\section{Author Contributions}

TL, KK and LK were involved in patient care and writing the manuscript. EJ was involved in the morphological investigation and writing the manuscript. SFL was involved in the histopathological analysis and the revision of the manuscript. AL, AS and MP were involved in cardiovascular imaging and writing the manuscript. TS was involved in writing and revision of the manuscript. Each author has reviewed the final version of the manuscript and approved it for publication.

\section{References}

1. Burke AP, Virmani R. Tumors of the heart and great vessels. In: Rosai J, Sobin LH, eds. Atlas of tumor pathology. Third series, fascicle 16. Armed Forces Institute of Pathology. Washington, DC, 1996.

2. Patel J, Sheppard MN. Pathological study of primary cardiac and pericardial tumours in a specialist UK Centre: surgical and autopsy series. Cardiovasc Pathol. 2010;19(6):343-352.

3. Burke AP, Cowan D, Virmani R. Primary sarcomas of the heart. Cancer. 1992;69(2):387-395.

4. Roberts WC. Primary and secondary neoplasms of the heart. Am J Cardiol. 1997;80(5):671-682.

5. Sparrow PJ, Kurian JB, Jones TR, Sivananthan MU. MR imaging of cardiac tumours. Radiographics. 
1999; 19:1421-1434.

6. Lurito KJ, Martin T, Cordes T. Right atrial primary cardiac osteosarcoma. Pediatr Cardiol. 2002;23(4):462-465.

7. Dell'Amore A, Asadi N, Caroli G, Dolci G, Bini A, Stella F. Recurrent primary cardiac osteosarcoma: a case report and literature review. Gen Thorac Cardiovasc Surg. 2014;62(3):175-180.

8. Centofanti P, Di Rosa E, Deorsola L, Dato GM, Patane F, La Torre M, Barbato L, et al. Primary cardiac tumors: early and late results of surgical treatment in 91 patients. Ann Thorac Surg. 1999;68(4):1236-1241.

9. Platonov MA, Turner AR, Mullen JC, Noga M, Welsh RC. Tumour on the tricuspid valve: metastatic osteosarcoma and the heart. Can J Cardiol. 2005;21(1):63-67.

10. Elasfar A, Khalifa A, Alghamdi A, Khalid R, Ibrahim M, Kashour T. Asymptomatic metastatic osteosarcoma to the right ventricle: Case report and review of the literature. J Saudi Heart Assoc. 2013;25(1):39-42.

11. Maleki M, Mozaffari K, Givtaj N, Tatina A, Bahadorian B. Metastatic osteosarcoma and heart: a rare involvement in an unusual cardiac location. Heart Asia. 2013;5(1):120121.

12. Yedururi S, Morani AC, Gladish GW, Vallabhaneni S, Anderson PM, Hughes D, Wang WL, et al. Cardiovascular involvement by osteosarcoma: an analysis of 20 patients. Pediatr Radiol. 2016;46(1):21-33.

13. Ye Z, Shi H, Peng T, Han A. Clinical and pathological features of high grade primary cardiac osteosarcoma. Interact Cardiovasc Thorac Surg. 2011;12(1):94-95.

14. Aguilar CA, Donet JA, Galarreta CI, Yabar A. A primary cardiac osteosarcoma: Case report and review of the literature. J Cardiol Cases. 2013;7(2):e29-e33.

15. Galas A, Hryniewiecki T, Szymanski P. An 82-year-old woman with a cardiac mass. Heart. 2016;102(11):881.

16. Liao JN, Chen IM, Yang AH, Yu WC. A primary cardiac sarcoma spreading along the pacing leads of a permanent pacemaker. J Am Coll Cardiol. 2012;59(16):1487.

17. Agaimy A, Rosch J, Weyand M, Strecker T. Primary and metastatic cardiac sarcomas: a 12-year experience at a German heart center. Int J Clin Exp Pathol. 2012;5(9):928-938.

18. Wang JG, Liu B, Gao H, Li YJ, Zhao P, Liu XP. Primary cardiac osteosarcoma. Heart Lung Circ. 2016;25(7):698704.

19. Sheppard MN, Mohiaddin R. Tumors of the heart. Future Cardiol. 2010;6(2):181-193.

20. Iyigun T, Ciloglu U, Ariturk C, Civelek A, Tosun R. Recurrent cardiac metastasis of primary femoral osteosarcoma: a case report. Heart Surg Forum. 2010;13(5):E333335.

21. Tiemann K, Stypmann J, Landeta F, Wiebe S, Schafers M, Schober O, Weckesser M, et al. Three-dimensional echo- cardiography and 153Sm-EDTMP SPECT/CT in extensive cardiac metastases from osteosarcoma. Eur J Nucl Med Mol Imaging. 2010;37(12):2406.

22. Navalkele P, Jones SM, Jones JK, Salazar JD, Toy PC, Iyer RV, Herrington B. Osteosarcoma tumor thrombus: a case report with a review of the literature. Tex Heart Inst J. 2013;40(1):75-78.

23. Schneiderman H, Fordham EW, Goren CC, McCall AR, Rosenberg MS, Rozek S. Primary cardiac osteosarcoma: multidisciplinary aspects applicable to extraskeletal osteosarcoma generally. CA Cancer J Clin. 1984;34(2):110117.

24. Forslund T, Melin J, Seppa A. Primary osteosarcoma of the right heart ventricle and atrium; a case report. Clin Med Oncol. 2008;2:43-46.

25. Fujino S, Miyoshi N, Ohue M, Noura S, Hamamoto S, Oshima K, Araki N, et al. Primary osteosarcoma of the heart with long-term survival: A case report of laparoscopic resection of a metastatic sarcoma in the intestine. Oncol Lett. 2014;8(4):1599-1602.

26. Hashimoto W, Hashizume K, Ariyoshi T, Taniguchi S, Miura T, Kinoshita N, Eishi K. Primary cardiac osteosarcoma with imaging that revealed no calcification. Gen Thorac Cardiovasc Surg. 2011;59(3):184-186.

27. Stark P, Smith DC, Watkins GE, Chun KE. Primary intrathoracic extraosseous osteogenic sarcoma: report of three cases. Radiology. 1990;174(3 Pt 1):725-726.

28. Sordillo PP, Hajdu SI, Magill GB, Golbey RB. Extraosseous osteogenic sarcoma. A review of 48 patients. Cancer. 1983;51(4):727-734.

29. Korkmaz O, Goksel S, Egilmez HR, Berkan O. Extraskeletal chondroblastic osteosarcoma in the left atrium. Interact Cardiovasc Thorac Surg. 2014;19(6):1077-1079.

30. Sakashita H, Miyata M, Miyamoto H, Kurumaya H. A case of quadruple cancer, including triple cancers in the head and neck region. J Oral Maxillofac Surg. 1996;54(4):501505.

31. Orlova PN, Parshikova SM. [Case of rhabdomyosarcoma of the heart associated with adenocarcinoma of the lungs and carcinoid of the large intestine]. Klin Med (Mosk). 1971;49(6):139-143.

32. Fukuoka M, Hagiwara M, Shimoshige S, Hirata A, Adachi T, Komura H, Shoji T, et al. Primary leiomyosarcoma of the heart subsequent to double carcinomas of the thyroid and lung. Heart Vessels. 2000;15(2):100-102.

33. Liu B, Sun W, Wang K. A successful insertion of PICC in patient with cardiac angiosarcoma and neoplasty of right atrium and pacemaker: A case report. Medicine (Baltimore). 2017;96(51):e9225.

34. Hong JT, Son DJ, Lee CK, Yoon DY, Lee DH, Park MH. Interleukin 32, inflammation and cancer. Pharmacol Ther. 2017;174:127-137. 